\title{
Prevalência e fatores associados à dor e ao histórico de lesões musculoesqueléticas em skatistas
}

\section{Prevalence and Factors Associated with Pain and History of Musculoskeletal Injuries in Skateboarders}

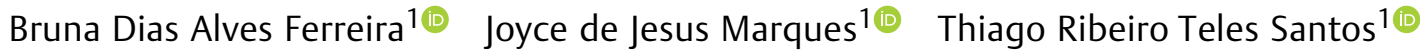 \\ ${ }^{1}$ Centro Universitário de Belo Horizonte, Belo Horizonte, MG, Brasil \\ Endereço para correspondência Thiago Ribeiro Teles Santos, PhD, \\ Rev Bras Ortop 2021;56(5):567-573.

\begin{abstract}
Resumo
Palavras-chave

- dor

- epidemiologia

- esportes

- sistema musculoesquelético/ lesões

- skate

Objetivo (a) Identificar a prevalência de dor musculoesquelética atual e lesão no último ano e (b) verificar a associação da presença de dor atual e da história de lesão com características demográficas, da prática esportiva e da saúde em skatistas.

Método O presente estudo observacional transversal foi realizado com 64 skatistas que responderam a um questionário abordando características demográficas, da prática esportiva e da saúde. O teste exato de Fisher verificou a associação dessas características com a presença de dor atual e com o relato de lesão no último ano. $O$ teste de qui-quadrado verificou se havia diferença entre a distribuição observada e a esperada para o segmento corporal relatado com dor e com histórico de lesão, e com o tipo de lesão.

Resultados A prevalência de dor foi de $82,8 \%$, sendo a frequência observada maior do que a esperada $(p<0,01)$ no joelho, no tornozelo, na região lombossacra e no pé. A presença de dor foi associada à faixa etária $(p=0,05)$ e a tratamento fisioterápico prévio $(p<0,01)$. A prevalência de lesão no último ano foi de $68,8 \%$, sendo a frequência observada maior do que a esperada $(p<0,01)$ no joelho, no tornozelo, no punho, na mão, no pé e no ombro. Entorse e fratura apresentaram uma frequência observada maior do que a esperada $(p<0,01)$. O histórico de lesão foi associado ao uso de equipamento de proteção $(p=0,01)$, cirurgia prévia $(p=0,02)$ e tratamento fisioterápico $(p=0,03)$.

Conclusão A prática de skate apresenta uma prevalência alta de dor e de lesões no último ano. Os achados podem contribuir com o planejamento de estratégias preventivas.
\end{abstract}

recebido

05 de Janeiro de 2021

aceito

08 de Março de 2021
DOI https://doi.org/

10.1055/s-0041-1731655. ISSN 0102-3616. (c) 2021. Sociedade Brasileira de Ortopedia e Traumatologia. All rights reserved.

This is an open access article published by Thieme under the terms of the Creative Commons Attribution-NonDerivative-NonCommercial-License, permitting copying and reproduction so long as the original work is given appropriate credit. Contents may not be used for commercial purposes, or adapted, remixed, transformed or built upon. (https://creativecommons.org/ licenses/by-nc-nd/4.0/)

Thieme Revinter Publicações Ltda., Rua do Matoso 170, Rio de Janeiro, RJ, CEP 20270-135, Brazil 


\begin{abstract}
Objective (a) To identify the prevalence of current musculoskeletal pain and injury in the last year and (b) to verify the association of the presence of current pain and the history of injury with demographic, sports, and health characteristics in skateboarders. Method A cross-sectional observational study was conducted with 64 skateboarders who answered a questionnaire addressing demographic, sports practice, and health characteristics. The Fisher exact test verified the association of these characteristics with the presence of current pain and with the report of injury in the last year. The chisquared test verified whether there was a difference between the observed and expected distribution for the body segment reported with pain and history of injury, and with the type of injury.

Results The prevalence of pain was of $82.8 \%$, and the observed frequency was higher than expected $(p<0.01)$ in the knee, the ankle, the lumbosacral region, and the foot. The presence of pain was associated with age $(p=0.05)$ and with whether the patient had already undergone physical therapy treatment $(p<0.01)$. The prevalence of injury in the last year was of $68.8 \%$, and the frequency observed was higher than expected

\section{Keywords}

- pain

- epidemiology

- sports

- musculoskeletal system/injuries

- skate $(p<0.01)$ in the knee, the ankle, the wrist, the hand, the foot, and the shoulder. Sprain and fracture showed a higher frequency than expected $(p<0.01)$. The history of injury was associated with the use of protective equipment $(p=0.01)$, having already undergone surgery $(p=0.02)$, and physical therapy treatment $(p=0.03)$.

Conclusion The practice of skateboarding presents a high prevalence of pain and injuries in the last year. The findings may contribute to the planning of preventive strategies.
\end{abstract}

\section{Introdução}

Dados de setores de emergência indicam um grande número de lesões devidas à prática de skate. ${ }^{1-3}$ Este esporte demanda a geração de grande força propulsiva e capacidade de lidar com forças de impacto de alta magnitude. ${ }^{4}$ A alta demanda ao sistema musculoesquelético pode contribuir não só com o desenvolvimento de lesões, mas também com o quadro de dor. Poucos estudos investigaram a dor musculoesquelética em skatistas. ${ }^{5}$ Este quadro pode alterar o desempenho do atleta e, assim, impactar sua prática esportiva. ${ }^{6}$ Apesar disso, observa-se que, comumente, a prática do skate não possui abordagem preventiva, como ocorre em outros esportes. Desta forma, a ampliação do entendimento das lesões e dores musculoesqueléticas em skatistas pode contribuir com o embasamento necessário para o planejamento de ações preventivas.

Características demográficas, da prática esportiva e da saúde podem estar associadas às lesões e dores musculoesqueléticas em skatistas. Características demográficas como sexo e idade do skatista são indicadas em estudos sobre lesões, com sugestão que os mais jovens tendem a se lesionar mais frequentemente. ${ }^{2,3}$ Enquanto estudos indicam que as lesões são comuns em skatistas do sexo masculino, ${ }^{2,3}$ um estudo recente sugere que entorses podem ser mais frequentes no sexo feminino. ${ }^{7}$ Características da prática esportiva, como nível de experiência e uso de equipamentos de proteção, também são comumente levantadas em estudos que descrevem o perfil de lesão. ${ }^{7-9}$ Características de atenção à saúde, como realizar atividade preventiva e ter assistência de profissional da saúde, são menos estudadas, apesar de serem relacionadas a lesões no esporte. ${ }^{10}$ Assim, estudos sobre lesões e dores musculoesqueléticas em skatistas devem considerar as possíveis associações com características demográficas, da prática esportiva e da saúde.

O skate foi recentemente reconhecido como esporte olímpico e, assim, há necessidade de ampliar as evidências sobre o perfil de lesões. ${ }^{8} \mathrm{O}$ estabelecimento das características da lesão e de sua extensão é entendido no campo da medicina esportiva como o primeiro passo para o planejamento de ações preventivas. ${ }^{11}$ Portanto, os objetivos do presente estudo foram (a) identificar a prevalência de dor musculoesquelética atual e lesão no último ano em skatistas, (b) verificar a associação da presença de dor atual e de lesão no último ano com as características demográficas, da prática esportiva e da saúde de skatistas.

\section{Métodos}

Foi realizado um estudo observacional transversal. A amostra foi recrutada por conveniência, em competições e pistas de skate na região metropolitana de Belo Horizonte, MG. Para participar do presente estudo, o voluntário deveria ser praticante de skate. Participantes menores de idade deveriam obrigatoriamente ter o consentimento do responsável. Foram excluídos os voluntários que praticassem o esporte menos de uma vez por semana. O presente estudo foi aprovado pelo Comitê de Ética em Pesquisa da instituição (CAAE 21230919.9.0000.5093) e todos os participantes assinaram o termo de consentimento livre e esclarecido. 
Tabela 1 Características demográficas

\begin{tabular}{|l|l|}
\hline & $\boldsymbol{n}(\%)$ \\
\hline Idade & \\
\hline $11-16$ anos & $3(4,7 \%)$ \\
\hline $17-22$ anos & $17(26,6 \%)$ \\
\hline $23-28$ anos & $16(25,0 \%)$ \\
\hline $29-34$ anos & $11(17,2 \%)$ \\
\hline $35-40$ anos & $8(12,5 \%)$ \\
\hline $41-46$ anos & $6(9,4 \%)$ \\
\hline$>46$ anos & $3(4,7 \%)$ \\
\hline Sexo & \\
\hline Masculino & $53(82,8 \%)$ \\
\hline Feminino & $11(17,2 \%)$ \\
\hline
\end{tabular}

Um questionário foi aplicado por 2 pesquisadores durante o $2^{\circ}$ semestre de 2019 , contendo perguntas agrupadas em: características demográficas, da prática esportiva e da saúde. As características demográficas investigadas foram idade e sexo. As características da prática esportiva investigadas foram: modalidade e categoria descritas pela Confederação Brasileira de Skate, ${ }^{12}$ tempo de prática esportiva, frequência de prática semanal, quantidade de horas por dia de prática, objetivo da prática (diversão, competição ou ambos) e se o skatista faz uso de equipamento de proteção durante a prática. As características de saúde investigadas foram: presença de dor no momento de aplicação do questionário e, caso afirmativo, a parte do corpo na qual sentia dor; histórico de lesão musculoesquelética no último ano e, caso afirmativo, a parte do corpo lesionada e o tipo de lesão. A lesão foi definida como qualquer queixa física devido à prática de skate que resultou em modificação da prática esportiva ou não participação em treino ou competição. 7,13 Para a categorização da parte do corpo que sentia dor e que estava lesionada, assim como do tipo de lesão, seguiu-se a recomendação do International Olympic Committee Injury and Illness Epidemiology Consensus Group. ${ }^{14}$ Além disso, foi investigado se o participante já havia realizado alguma cirurgia ou tratamento fisioterápico decorrente de lesão gerada pela prática do skate, assim como se realizava alguma atividade que considerasse preventiva.

\section{Análise Estatística}

A prevalência de dor no momento de aplicação do questionário, assim como do histórico de lesão no último ano, foi calculada. $O$ teste exato de Fisher foi realizado para verificar a associação entre a presença de dor, assim como presença de lesão no último ano, e as variáveis investigadas no questionário. Na presença de associação significativa, análise do resíduo ajustado foi realizada para identificar qual célula da tabela de contingência realizou uma contribuição significativa para o resultado. Nesta análise, se o valor do resíduo ajustado é além de $\pm 1,96$, há indicação de que o número de casos na célula da tabela de contingência foi diferente do esperado. 0 teste de qui-quadrado goodness of fit foi realizado para verificar se a distribuição observada era diferente da esperada nas respostas relativas ao segmento do corpo com dor e com história de lesão, assim como ao tipo de lesão. O nível de significância $(\alpha)$ de 0,05 foi estabelecido para todos os testes.

\section{Resultados}

Sessenta e quatro skatistas participaram do presente estudo. As características demográficas estão apresentadas na - Tabela 1. A frequência observada em cada resposta relativa às características da prática esportiva e de saúde estão apresentadas nas - Tabelas 2 e 3.

\section{Dor atual}

A prevalência de dor no momento da aplicação do questionário foi de $82,8 \%$ (53 skatistas). Os segmentos corporais relatados com dor estão apresentados na - Tabela 4 . 0 teste de qui-quadrado revelou que a distribuição das respostas não era uniforme e, portanto, era diferente da esperada em relação ao segmento corporal com dor $\left(X^{2}[10]=74,91\right.$; $p<0,01)$. O joelho, o tornozelo, a região lombossacra e o pé apresentaram frequência observada maior do que a esperada, enquanto os demais segmentos apresentaram frequência menor do que a esperada.

A presença de dor foi associada à faixa etária $(p=0,05)$. A análise do resíduo ajustado indicou que, na categoria de entre 11 e 16 anos, houve maior contribuição para essa associação daqueles que não apresentavam dor $(Z=3,9)$ e menor contribuição daqueles que apresentavam dor $(Z=-$ $3,9)$. A presença de dor também foi associada a tratamento fisioterápico prévio $(p<0,01)$. A análise de resíduo ajustado indicou que entre aqueles que não realizaram tratamento, houve maior contribuição para esta associação daqueles que não possuíam dor $(Z=4,1)$, e menor contribuição daqueles que possuíam dor $(Z=-4,1)$. Entre aqueles que realizaram tratamento fisioterápico, houve maior contribuição daqueles que sentem dor $(Z=4,1)$ e menor daqueles que não sentem dor $(Z=-4,1)$.

Não foi observada associação entre a presença de dor e as seguintes variáveis: sexo $(p=0,67)$, modalidade do skate $(p=0,23)$, tempo de prática esportiva $(p=0,67)$, frequência de prática semanal $(p=0,74)$, horas por dia de prática $(p=0,20)$, objetivo da prática $(p=0,21)$, categoria do skatista $(p=0,87)$, uso de equipamento de proteção $(p=0,47)$, história de lesão no último ano $(\mathrm{p}=0,30)$, história de cirurgia $(p=1,00)$, e se o skatista realizou alguma atividade que considerasse que prevenisse lesões $(p=1,00)$.

\section{Histórico de lesão no último ano}

A prevalência de lesão no último ano foi de $68,8 \%$ (44 skatistas). Os segmentos corporais relatados com história de lesão e o tipo de lesão estão apresentados nas - Tabelas 5 e $\mathbf{6}$, respectivamente. $\mathrm{O}$ teste de qui-quadrado revelou que a distribuição das respostas não era uniforme e, portanto, esta foi diferente da esperada quanto ao segmento corporal $\left(X^{2}[12]=48,94\right.$; $p<0,01)$ e ao tipo de lesão $\left(X^{2}[6]=44,39 ; p<0,01\right)$. A 
Tabela 2 Características da prática esportiva

\begin{tabular}{|l|l|l|l|}
\hline & $\mathbf{n}(\%)$ & & $n$ (\%) \\
\hline Modalidade & & Frequência semanal de treinos & \\
\hline Banks & $1(1,6 \%)$ & $1 \mathrm{x}$ & $7(10,9 \%)$ \\
\hline Bowl & $3(4,7 \%)$ & $2 \mathrm{x}$ & $12(18,8 \%)$ \\
\hline Overall & $9(14,1 \%)$ & $3 \mathrm{x}$ & $16(25,0 \%)$ \\
\hline Park & $2(3,1 \%)$ & $4 \mathrm{x}$ & $9(14,1 \%)$ \\
\hline Street & $46(71,9 \%)$ & $5 \mathrm{x}$ & $11(17,2 \%)$ \\
\hline Vertical & $3(4,7 \%)$ & $6 \mathrm{x}$ & $5(7,8 \%)$ \\
\hline Categoria & & $7 \mathrm{x}$ & $4(6,3 \%)$ \\
\hline Amador & $30(46,9 \%)$ & Horas por treino & \\
\hline Grand Legend & $1(1,6 \%)$ & $<1 \mathrm{~h}$ & $1(1,6 \%)$ \\
\hline Grand Master & $1(1,6 \%)$ & $1-2 \mathrm{~h}$ & $22(34,4 \%)$ \\
\hline Iniciante & $13(20,3 \%)$ & $3-4 \mathrm{~h}$ & $25(39,1 \%)$ \\
\hline Legend & $4(6,3 \%)$ & $>5 h$ & $16(25,0 \%)$ \\
\hline Master & $4(6,3 \%)$ & Objetivos da prática & \\
\hline Mirim & $1(1,6 \%)$ & Competição & $1(1,6 \%)$ \\
\hline Profissional & $9(14,1 \%)$ & Diversão & $29(45,3 \%)$ \\
\hline Vintage & $1(1,6 \%)$ & Diversão e Competição & $34(53,1 \%)$ \\
\hline Tempo de prática esportiva & & Uso de equipamento de proteção & \\
\hline$<1$ ano & $2(3,1 \%)$ & Não & $38(59,4 \%)$ \\
\hline $1-5$ anos & $15(23,4 \%)$ & Sim & $15(23,4 \%)$ \\
\hline $6-10$ anos & $10(15,6 \%)$ & Só em competições & $11(17,2 \%)$ \\
\hline$>10$ anos & $37(57,8 \%)$ & & \\
\hline
\end{tabular}

frequência observada foi maior do que a esperada no joelho, no tornozelo, no punho, na mão, no pé e no ombro, enquanto nos demais segmentos, ela foi menor do que a esperada. Já o tipo de lesão apresentou frequência observada maior do que a esperada para entorse, fratura e para aqueles que não conseguiram especificar, enquanto nos demais tipos a frequência foi menor do que a esperada.

A presença de lesão no último ano foi associada ao uso de equipamento de proteção $(p=0,01)$. A análise do resíduo ajustado indicou que, na resposta uso somente em compe-

Tabela 3 Características relacionadas à saúde

\begin{tabular}{|l|l|}
\hline & $\boldsymbol{n}(\%)$ \\
\hline História de cirurgia devido à lesão por esporte & \\
\hline Não & $54(84,4 \%)$ \\
\hline Sim & $10(15,6 \%)$ \\
\hline História de tratamento fisioterápico & \\
\hline Não & $28(43,8 \%)$ \\
\hline Sim & $36(56,3 \%)$ \\
\hline Realiza atividade que considera como preventiva & \\
\hline Não & $22(34,4 \%)$ \\
\hline Sim & $42(65,6 \%)$ \\
\hline
\end{tabular}

tição, houve maior contribuição para essa associação daqueles que não possuíam histórico de lesão $(Z=3,3)$ e menor contribuição daqueles que possuíam histórico de lesão $(Z=-$ $3,3)$. Houve também associação entre o histórico de lesão e história de cirurgia devido à lesão por skate $(p=0,02)$. A análise do resíduo ajustado indicou que entre aqueles que não relataram histórico de cirurgia, houve maior contribuição para associação daqueles que não tinham histórico de lesão $(Z=2,3)$ e menor contribuição daqueles com histórico de lesão $(Z=-2,3)$. Já entre aqueles que relataram histórico de cirurgia, houve maior contribuição para associação daqueles com história de lesão $(Z=2,3)$ e menor contribuição daqueles sem história de lesão $(Z=-2,3)$.

A presença de lesão no último ano também foi associada a tratamento fisioterápico prévio $(p=0,03)$. A análise do resíduo ajustado indicou que entre aqueles que não realizaram fisioterapia, houve maior contribuição para associação daqueles que não tinham histórico de lesão $(Z=2,3)$, e menor contribuição daqueles com histórico de lesão $(Z=-2,3)$. Já entre aqueles que realizaram fisioterapia, houve maior contribuição para associação daqueles com histórico de lesão $(Z=2,3)$, e menor contribuição daqueles sem histórico de lesão $(Z=-2,3)$.

Não foi observada associação entre a presença de lesão no último ano e as seguintes variáveis: faixa etária $(p=0,83)$, sexo $(p=0,73)$, modalidade do skate $(p=0,79)$, tempo de 
Tabela 4 Segmento corporal relatado como com dor no momento de aplicação do questionário, ordenado do segmento mais frequente para o menos frequente

\begin{tabular}{|l|l|}
\hline Segmento corporal & $\boldsymbol{n}(\%)$ \\
\hline Joelho & $32(23,0 \%)$ \\
\hline Tornozelo & $24(17,3 \%)$ \\
\hline Coluna lombossacra & $23(16,5 \%)$ \\
\hline Pé & $15(10,8 \%)$ \\
\hline Punho/Mão & $10(7,2 \%)$ \\
\hline Ombro & $10(7,2 \%)$ \\
\hline Perna & $8(5,8 \%)$ \\
\hline Quadril & $7(5,0 \%)$ \\
\hline Braço & $4(2,9 \%)$ \\
\hline Coxa & $4(2,9 \%)$ \\
\hline Cotovelo & $2(1,4 \%)$ \\
\hline Total & $139(100,0 \%)$ \\
\hline
\end{tabular}

Tabela 5 Segmento corporal com histórico de lesão no último ano, ordenado do segmento mais frequente para o menos frequente

\begin{tabular}{|l|l|}
\hline Segmento corporal & $\boldsymbol{n}(\%)$ \\
\hline Joelho & $14(21,9 \%)$ \\
\hline Tornozelo & $13(20,3 \%)$ \\
\hline Punho/Mão & $8(12,5 \%)$ \\
\hline Pé & $7(10,9 \%)$ \\
\hline Ombro & $6(9,4 \%)$ \\
\hline Coluna lombossacra & $4(6,3 \%)$ \\
\hline Quadril/Virilha & $3(4,7 \%)$ \\
\hline Cabeça & $3(4,7 \%)$ \\
\hline Perna & $2(3,1 \%)$ \\
\hline Coxa & $1(1,6 \%)$ \\
\hline Tronco & $1(1,6 \%)$ \\
\hline Cotovelo & $1(1,6 \%)$ \\
\hline Antebraço & $1(1,6 \%)$ \\
\hline Braço & $1(1,6 \%)$ \\
\hline Total & $64(100,0 \%)$ \\
\hline
\end{tabular}

prática esportiva $(p=0,97)$, frequência de prática semanal $(p=0,16)$, horas por dia de prática $(p=0,47)$, objetivo da prática $(p=0,20)$, categoria do skatista $(p=0,86)$ e realização de alguma atividade que o skatista considerasse que prevenisse lesões $(p=0,58)$.

\section{Discussão}

O presente estudo identificou a prevalência de dor musculoesquelética atual e de lesão esportiva no último ano em skatistas e também verificou a associação da presença de dor
Tabela 6 Tipo de lesão sofrida no último ano, ordenado do tipo mais frequente para o menos frequente

\begin{tabular}{|l|l|}
\hline Tipo de lesão & $\boldsymbol{n}(\%)$ \\
\hline $\begin{array}{l}\text { Entorse articular (ruptura ligamentar ou } \\
\text { episódio de instabilidade aguda) }\end{array}$ & $22(42,3 \%)$ \\
\hline Fratura & $11(21,2 \%)$ \\
\hline Lesão em cartilagem & $4(7,7 \%)$ \\
\hline Lesão muscular & $3(5,8 \%)$ \\
\hline Tendinopatia & $2(3,8 \%)$ \\
\hline Lesão por estresse em osso & $1(1,9 \%)$ \\
\hline Não soube informar & $9(17,3 \%)$ \\
\hline Total & $52(100,0 \%)$ \\
\hline
\end{tabular}

Nota: Categorias seguem as recomendadas pelo International Olympic Committe Injury and IIIness Epidemiology Consensus Group, ${ }^{14}$ em que entorse inclui ruptura parcial ou completa assim como lesões não específicas de ligamentos e cápsula articular, luxação e subluxação articular; fratura é uma lesão traumática que inclui fratura por avulsão e dentária; lesão em cartilagem inclui lesão meniscal, labral, da cartilagem articular e osteocondral; lesão muscular inclui estiramento e ruptura; tendinopatia inclui o paratendão, bursa relacionada, fasciopatia, ruptura parcial, subluxação tendínea e entesopatia; lesão por estresse em osso inclui edema da medula óssea, fratura por estresse e periostite.

e lesão com as características demográficas, da prática esportiva e da saúde. A prevalência de dor foi alta e mais frequente no joelho, no tornozelo, na região lombossacra e no pé. A comparação destes achados com outros é restrita devido ao número limitado de estudos sobre dor musculoesquelética em skatistas. Os resultados corroboram parcialmente outro estudo que não identificou associação da prática de skate com dor em três segmentos corporais investigados: ombro, coluna cervical e lombar. ${ }^{5}$ Desta forma, a alta prevalência de dor, principalmente nos membros inferiores, reforça o entendimento de que o skate é um esporte que oferece grande sobrecarga ao sistema musculoesquelético.

A categoria entre 11 e 16 anos apresentou menor associação com o relato de dor. Outros estudos indicam maior hospitalização nessa faixa etária e hipotetizam que o motivo seria a pior coordenação motora entre os jovens. ${ }^{8,15}$ Possivelmente, os skatistas mais jovens procuram atendimento por traumas ao invés de lesões por overuse. Além disso, a categoria entre 11 e 16 anos foi uma das que apresentaram menor quantidade de skatistas recrutados. Como a amostragem utilizada foi não-probabilística, os skatistas recrutados podem apresentar alguma característica da população super-representada ou sub-representada. ${ }^{16}$ Assim, futuros estudos podem verificar se alguma outra característica influenciou a associação identificada.

A presença de dor teve ainda associação com tratamento fisioterápico prévio. Devido ao desenho transversal do presente estudo, não é possível verificar se a dor estava presente antes ou se surgiu após o tratamento fisioterápico. A associação identificada pode sugerir que os fatores relacionados à presença de dor sejam similares ao da condição musculoesquelética que levou o skatista a ser tratado por fisioterapeuta. 
A prevalência de lesões no último ano também foi alta, mas inferior às encontradas em outros estudos. ${ }^{7,17}$ As lesões ocorreram mais frequentemente no joelho, no tornozelo, no punho, na mão, no pé e no ombro. Este resultado corrobora estudos que indicam que a maioria das lesões ocorre nos membros inferiores $^{7,15,17}$ e superiores. ${ }^{8,18-20}$ Nos membros inferiores, as lesões no joelho são geralmente o resultado de impacto direto, de sobrecarga (por exemplo, dor patelofemoral), assim como de lesões do menisco e do ligamento cruzado. ${ }^{18}$ Estas lesões, provavelmente, são o resultado das características dos gestos esportivos, os quais demandam muito do mecanismo extensor do joelho. ${ }^{20,21}$ Estes gestos envolvem ainda salto (por exemplo, ollie) e mudanças de direção que incluem movimentos rotacionais no joelho (por exemplo, body varial), padrão tipicamente associado a lesões ligamentares e de menisco. ${ }^{22,23}$ A literatura indica ainda que um dos mecanismos de lesão de tornozelo é quando ele fica preso entre a prancha do skate e o chão ou, ainda, por colisão com veículos. ${ }^{8}$ Nos membros superiores, há destaque para a região do punho e da mão, ${ }^{8,19,24}$ comumente lesionados devido à queda sobre 0 membro superior com o cotovelo e punho estendidos. ${ }^{8,9}$ Além disso, as lesões do tipo entorse e fratura foram as mais frequentes, o que corrobora outros achados. ${ }^{2,7,8,15,20,24} \mathrm{~A}$ literatura sugere que as entorses ocorrem frequentemente no tornozelo devido às manobras realizadas. ${ }^{23}$ Assim, o histórico de lesão reforça que a prática de skate possui um perfil de lesões graves ao sistema musculoesquelético.

A ausência de lesão no último ano foi associada ao uso de equipamento de proteção durante competições. Este resultado corrobora a recomendação do uso destes equipamentos como estratégia preventiva. ${ }^{8}$ Apesar disso, muitos skatistas optam por não usar equipamento de proteção. ${ }^{9}$ Desta forma, apesar de argumentos comuns para o não uso, como desconforto e aparência, ${ }^{8}$ os resultados reforçam que o uso deve ser encorajado. Destaca-se que os equipamentos de proteção podem não ser suficientes para prevenir todas as lesões, como as de alta magnitude de carga (por exemplo, entorses severas de joelho).

Os skatistas que relataram ter realizado cirurgia e fisioterapia tiveram maior associação com a história de lesão. Estes achados reforçam a interpretação de que, possivelmente, os fatores que levaram ao desenvolvimento de lesão favoreceram também o surgimento da condição musculoesquelética que levou o skatista a procurar assistência em saúde. Além disso, não foi identificada associação entre a realização de atividade que o skatista considerasse preventiva e a presença de lesão ou dor. Apesar disso, estudos indicam que treino para a melhora do desempenho físico e da estabilidade articular dinâmica é uma estratégia para evitar e minimizar as lesões em skatistas. ${ }^{8} \mathrm{Um}$ dos motivos para não se observar essa associação podem ser crenças relativas a o que seria atividade preventiva, uma vez que foram observados relatos de que alongamento seria uma estratégia preventiva. $O$ baixo conhecimento do que é considerado atividade preventiva reforça a importância de maior suporte de profissionais de saúde por meio de ações educativas e de avaliações para rastreamento de possíveis fatores causais.

0 presente estudo apresenta algumas limitações. A maior parte dos skatistas recrutados era da modalidade street. Além disso, o registro de lesão pode apresentar viés de memória, ${ }^{16}$ no qual os skatistas tendem a reportar somente as lesões que mais impactaram sua prática. A restrição de lesões no último ano ao invés de em um período maior foi a estratégia adotada para minimizar este viés. Além disso, o registro de dor atual foi investigado por meio de questionário aplicado em pistas e competições de skate. A investigação desta variável em outros momentos poderia resultar em achados distintos. Assim, futuros estudos prospectivos que considerem maior participação de outras modalidades e a influência de diferentes contextos podem contribuir para o entendimento da dor e das lesões em skatistas.

\section{Conclusão}

A alta prevalência de dor atual e de lesão no último ano corroboram o entendimento de que o skatista está exposto a diversos riscos em sua prática. A dor foi mais frequente no joelho, no tornozelo, na região lombossacra e no pé, estando associada com faixa etária e com tratamento fisioterápico prévio. A lesão no último ano foi mais frequente no joelho, no tornozelo, no punho, na mão, no pé e no ombro. Os tipos de lesão mais comuns foram entorse e fratura. A história de lesão foi associada ao uso de equipamento de proteção a cirurgia e tratamento fisioterápico prévios.

\section{Nota}

Trabalho desenvolvido no Centro Universitário de Belo Horizonte (UniBH), Belo Horizonte, MG, Brasil

\section{Suporte Financeiro}

Não houve suporte financeiro de fontes públicas, comerciais, ou sem fins lucrativos.

\section{Conflito de Interesses}

Os autores declaram não haver conflito de interesses.

\section{Referências}

1 Kyle SB, Nance ML, Rutherford GW Jr, Winston FK. Skateboardassociated injuries: participation-based estimates and injury characteristics. J Trauma 2002;53(04):686-690

2 Tuckel PS, Milczarski W, Silverman DG. Changing Incidence and Nature of Injuries Caused by Falls From Skateboards in the United States. Clin Pediatr (Phila) 2019;58(04):417-427

3 Zalavras C, Nikolopoulou G, Essin D, Manjra N, Zionts LE. Pediatric fractures during skateboarding, roller skating, and scooter riding. Am J Sports Med 2005;33(04):568-573

4 Frederick EC, Determan JJ, Whittlesey SN, Hamill J. Biomechanics of skateboarding: kinetics of the Ollie. J Appl Biomech 2006;22 (01):33-40

5 Auvinen JP, Tammelin TH, Taimela SP, Zitting PJ, Mutanen POA, Karppinen JI. Musculoskeletal pains in relation to different sport and exercise activities in youth. Med Sci Sports Exerc 2008;40 (11):1890-1900

6 Stevens CJ, Mauger AR, Hassmèn P, Taylor L. Endurance Performance is Influenced by Perceptions of Pain and Temperature: Theory, Applications and Safety Considerations. Sports Med 2018;48(03):525-537 
7 Rodríguez-Rivadulla A, Saavedra-García MÁ, Arriaza-Loureda R. Skateboarding Injuries in Spain: A Web-Based Survey Approach. Orthop J Sports Med 2020;8(03):2325967119884907

8 Feletti F, Brymer E. Pediatric and adolescent injury in skateboarding. Res Sports Med 2018;26(sup1):129-149

9 Fountain JL, Meyers MC. Skateboarding injuries. Sports Med 1996; 22(06):360-366

10 Valovich McLeod TC, Decoster LC, Loud KJ, et al. National Athletic Trainers' Association position statement: prevention of pediatric overuse injuries. J Athl Train 2011;46(02):206-220

11 van Mechelen W, Hlobil H, Kemper HC. Incidence, severity, aetiology and prevention of sports injuries. A review of concepts. Sports Med 1992;14(02):82-99

12 Confederação Brasileira de Skate (CBSK) Categorias [acessado em 26 de novembro de 2020]. Disponível em: http://www.cbsk. com.br/cms/normas/categorias/12

13 Fuller CW, Ekstrand J, Junge A, et al. Consensus statement on injury definitions and data collection procedures in studies of football (soccer) injuries. Br J Sports Med 2006;40(03):193-201

14 Bahr R, Clarsen B, Derman W, et al; International Olympic Committee Injury and Illness Epidemiology Consensus Group. International Olympic Committee Consensus Statement: Methods for Recording and Reporting of Epidemiological Data on Injury and Illness in Sports 2020 (Including the STROBE Extension for Sports Injury and Illness Surveillance (STROBE-SIIS)). Orthop J Sports Med 2020;8(02):2325967120902908
15 Forsman L, Eriksson A. Skateboarding injuries of today. Br J Sports Med 2001;35(05):325-328

16 Portney LG, Watkins MP. Foundations of Clinical Research: Applications to Practice. 3rd ed. Philadelphia, PA: F. A. Davis Company; 2015

17 Keilani M, Krall C, Lipowec L, Posch M, Komanadj TS, Crevenna R. Skateboarding injuries in Vienna: location, frequency, and severity. PM R 2010;2(07):619-624

18 Shuman KM, Meyers MC. Skateboarding injuries: An updated review. Phys Sportsmed 2015;43(03):317-323

19 Hunter J. The epidemiology of injury in skateboarding. Med Sport Sci 2012;58:142-157

20 Keays G, Dumas A. Longboard and skateboard injuries. Injury 2014;45(08):1215-1219

21 Candotti CT, Loss JF, Silva RE, et al. Lower limb force, power and performance in skateboarding: an exploratory study. Rev Bras Ciênc Esporte 2012;34(03):697-711

22 Mehl J, Diermeier T, Herbst E, et al. Evidence-based concepts for prevention of knee and ACL injuries. 2017 guidelines of the ligament committee of the German Knee Society (DKG). Arch Orthop Trauma Surg 2018;138(01):51-61

23 Fox AJS, Wanivenhaus F, Burge AJ, Warren RF, Rodeo SA. The human meniscus: a review of anatomy, function, injury, and advances in treatment. Clin Anat 2015;28(02):269-287

24 Bandzar S, Funsch DG, Hermansen R, Gupta S, Bandzar A. Pediatric Hoverboard and Skateboard Injuries. Pediatrics 2018;141(04): e20171253 